\title{
American and Exotic Options in a Market with Frictions
}

December 4, 2018

\begin{abstract}
In a market with frictions, bid and ask prices are described by sublinear pricing functionals, which can be defined recursively using coherent risk measures. We prove the convergence of bid and ask prices for various European and American possible path-dependent options, in particular plain vanilla, Asian, lookback and barrier options in a binomial model with transaction costs. We perform several numerical experiments to confirm the theoretical findings.

We apply the results to real market data of American options and compute an implied liquidity to describe the bid-ask spread. This method describes liquidity over time very well, compared to the classical approach of describing bid and ask prices by quoting bid and ask implied volatilities.

Keywords: Market with frictions, bid-ask spread, American options, barrier options, binomial model

JEL: G12, G13, D23, D52, C51
\end{abstract}

\section{Introduction}

In this article, we obtain closed-form solutions of bid and ask prices of European plain vanilla and barrier options in markets with frictions. Markets with frictions are markets with transaction costs. In contrast to complete financial markets without any imperfections where prices are obtained by a linear pricing rule, prices in markets with frictions can be described by sublinear pricing functionals, see Jouini (2000). Such pricing functionals may also describe prices in markets with additional or different kind of frictions than (proportional) transaction costs, like short sales costs or constrains, borrowing costs, taxes and other market imperfections, see Jouini and Kallal (2001), Koehl and Pham (2000), Bion-Nadal (2009) and references therein.

Jouini and Kallal $(1995,2001)$ and Jouini (2000) introduced an axiomatic approach to describe financial markets with frictions. They considered a finite time-horizon $T>0$ and a multiperiod economy, where investors can trade a riskless and a risky asset. Let $N$ be the number of trading periods in $[0, T]$. Jouini (2000) modelled the bid and ask price processes of the risk asset by 
adapted processes $0<S_{i}^{b} \leq S_{i}^{a}, i=0, . ., N$. They postulated the existence of a pricing functional $p$, which satisfies the following axioms: (i) $p$ is monotone, (ii) $p$ is sub-additive, (iii) $p$ is positively homogeneous, (iv) $p$ does not introduce arbitrage, (v) $p$ is lower-semi-continuous and (vi) for a future random cash flow $C, p(C)$ is less than or equal to the price of the smallest self-financing trading strategy dominating $C$. The ask price of a contingent claim $C$ is defined by $p(C)$, the bid-price is defined by $-p(-C)$, hence buying the contingent claim $C$ is the same as selling $-C$.

Jouini and Kallal (1995) showed that the market is arbitrage-free, if and only if there exist a measure $\mathbb{Q}$, equivalent to the physical measure $\mathbb{P}$, and a process $Z^{\mathbb{Q}}$, which is a martingale under $\mathbb{Q}$, such that $S^{b} \leq Z^{\mathbb{Q}} \leq S^{a}$. This leads to an easy construction of arbitrage-free financial markets with frictions: we take a complete frictionless market, the binomial model, where the risky underlying is described by some martingale $\left(S_{i}\right)_{i=0, \ldots, N}$ under the risk-neutral measure. Introducing sequence of dynamic coherent risk measures $\left(\rho_{i}\right)_{i=0, \ldots, N}$ and defining a pricing functional by $p_{i}():.=\rho_{i}(-$.$) , we introduce frictions into$ the market by defining the ask price process of the underlying by $\left(p_{i}\left(S_{N}\right)\right)$ and the bid price process by $\left(-p_{i}\left(-S_{N}\right)\right)$. The sequence $\left(p_{i}\right)$ based on a sequence of coherent risk measures fulfils axioms (i)-(vi).

\section{Contribution}

In a binomial-type model with frictions, bid and ask prices are recursively defined. For efficient numerical applications, we prove convergence of bid and ask prices of European and American plain vanilla and exotic options. We develop closed-form solutions for European plain vanilla and some barrier options and obtain in the limit an extended Black-Scholes formula with a new parameter $\gamma \geq 0$, which adjusts the dividend yield. The greater $\gamma$, the greater the bid-ask spread.

The limit bid or ask price of a possible path-dependent option is given by the Black-Scholes price of the option but on a stock with an adjusted dividend yield. Hence existing numerical methods, developed to price options in a Black-Scholes setting in classical finance, can also be used to compute bid and ask prices of such options. No new software need to be written to apply our formulas in financial institutions.

\section{Practical Relevance}

We think the main application area of our two-price formulas is the possibility of computing implicitly a parameter $\gamma$ such that given bid and ask market prices of an European or American plain vanilla option are exactly matched by our extended Black-Scholes formula.

This idea is comparable to the concept of implied volatility. In principle volatilities could be constant across strikes, maturities, and underlying assets, hence the preference by practitioners for quoting implied volatilities instead of (mid-)prices. Similarly the parameter $\gamma$ could be constant across all three 
dimensions, even though there are many non-linearities between the bid-ask spread and strikes, maturities, and underlying assets. It should therefore be beneficial to quote an implicitly computed $\gamma$ instead of the absolute bid-ask spread of a plain vanilla option. Indeed Corcuera et al. (2009) used a setting similar to our model, but in static time, and showed empirically that the liquidity dry up during the period 2007-2009 is described very well by the parameter $\gamma$. Our discrete time model makes it possible to analyse also path-dependent and American options.

Up to now, traders quote the difference between implied bid and ask volatilities to describe the current market liquidity of plain vanilla options. Both this heuristic method and our proposal of computing implicitly the parameter $\gamma$ have the advantage of using only present market data and of being extremely fast in terms of computational time, in both cases one has to invert the Black-Scholes formula. However, we show empirically that our model describes (il)liquidity of American plain vanilla options very well over time compared to the heuristic method of quoting implied bid and ask volatilities. In a static setting, a similar study has been done by Guillaume et al. (2018) for European plain vanilla options.

\section{Limitations}

Convergence is only proven for monotone payoffs, for example European or American plain vanilla, lookback, Asian and some barrier options (up-and-out put, down-and-out call, down-and-in put and up-and-in call).

The underlying is essentially modelled by a binomial model in discrete time and by geometric Brownian motion in continuous time. Hence the volatility is assumed to be constant over time and log-returns are assumed to be (approximately) normal distributed.

Future research need to be done to treat contingent claims which are not monotone with respect to the underlying, e.g. a barrier up-and-out call option and to generalize the market model replacing for example the constant volatility by a mean-reverting stochastic process.

\section{Literature Review}

In general, liquidity is effected by many factors like the ability of trading large quantities, by the speed, the cost and the price impact of the trade. Several measures have been developed in literature to capture some or all of these factors. Amihud (2002) defines the liquidity of a stock by the average of the ratio of absolute daily returns to volume, where the average is taken over a month. Acharya et al. (2005) developed a liquidity adjusted capital asset pricing model and measured liquidity using a normalized version of Amihud's liquidity measure. Liu (2006) analyse the relation between liquidity risk and asset pricing using a liquidity measure based on historic data. Goyenko et al. (2009) compared several well known liquidity measures using stock data from 1993 to 2005. 
In contrast to the above studies, which define (il)liquidity mainly using a historic time series of the stock, our model is well suited to be applied to an option surface and needs only present market data to compute the market implied liquidity parameter $\gamma$.

Recently, Madan and Cherny (2010) developed the conic finance theory. Our market model with frictions is connected to conic finance by the common approach of using recursively defined sublinear functionals to describe bid and ask prices. Indeed our discrete market model is closely related to discrete time conic finance models, where bid and ask prices are defined recursively using nonlinear expectations, see Leippold and Schärer (2017), Madan (2010), Madan, Pistorius and Schoutens $(2013,2017)$ and Madan and Schoutens (2012b). Time-consistent nonlinear expectations are connected with solutions to backward stochastic difference equations, see Cohen and Elliott (2010). See Bielecki et al. (2013) and Bielecki, Cialenco and Chen (2015) for a framework incorporating transaction costs in discrete time conic finance models.

Our work is related to Madan, Pistorius and Schoutens (2017) who showed in a general context that, under some technical conditions, an iterated spectral risk measure, which is a risk measure in a multiperiod setting based on distortion functions, converges to some g-expectation. A g-expectation is a non-linear expectation proposed by Peng (2004).

Relative to these papers our contribution is to proof convergence of bid and ask prices in a binomial-type model with frictions when the number of trading periods approaches infinity and to obtain closed-form solutions for bid and ask prices for plain vanilla and barrier options in the limit.

\section{Contents}

The remainder of the article is organized as follows. In Section 2, we introduce a discrete time-model for a market with frictions. In Section 3, we present the classical binomial model. In Section 4 we prove convergence of bid and ask prices for European and American possibly path-dependent options. In Section 5 , we apply the results to real market data. Section 6 concludes.

\section{The Formal Setup}

We make the following economic assumptions: we assume all investors have a finite time-horizon and trading can take place only finitely many times. There is a very liquid bank account and a risky-asset whose bid and ask prices can be described by binomial trees. There exists a pricing functional and bid and ask prices of a contingent claim can be computed via the pricing functional. At the end of the time-horizon, the bid-ask spread of all products is assumed to be zero.

Formally, we assume the following framework: Let $T>0$ be some timehorizon and $N \in \mathbb{N}$ be the number of trading periods, each trading period has length $\frac{T}{N}$. We introduce a frictionless market and extend it to a market with 
frictions using a pricing functional. Let the risky-asset

$$
\left(S_{i}\right)_{i=0,1, . ., N}
$$

be described by a nonnegative adapted stochastic process on a given filtered probability space

$$
\left(\Omega,\left(\mathcal{F}_{i}\right)_{i=0, \ldots, N}, \mathcal{F}, \mathbb{P}\right)
$$

satisfying the usual conditions. By

$$
B_{i}=(1+r)^{i}, i=0, . ., N
$$

we denote a risk-free bank account. We assume the market is arbitrage-free and denote by $\mathbb{Q}$ a risk-neutral measure, such that the discounted price process of the risky-asset is a $\mathbb{Q}$-martingale. The process $\left(S_{i}\right)$ describes the risky asset of the underlying frictionless market. In this section we assume that the interest rates are equal to zero, i.e. we work with discounted cash flows, and that the stock is not paying any dividends. Those assumptions are only made to keep the notation simple and will be relaxed in Section 3 and 4. Let

$$
L^{\infty}:=L^{\infty}(\Omega, \mathbb{Q}, \mathcal{F})
$$

be the set of $\mathcal{F}$-measurable bounded random variables with respect to the probability measure $\mathbb{Q}$ and $\left(\rho_{i}\right)_{i=0, \ldots, N}$ be a set of dynamic, time-consistent coherent risk measures being continuous from above

$$
\rho_{i}: L^{\infty} \rightarrow L_{i}^{\infty}:=L^{\infty}\left(\Omega, \mathbb{Q}, \mathcal{F}_{i}\right)
$$

We call the operator

$$
p_{i}(.):=\rho_{i}(-.), \quad i=0, \ldots, N
$$

a price functional. Then $\left(p_{i}\right)$ fulfils the following properties, see Föllmer and Schied (2011, Definition 11.1, Theorem 11.2. and Lemma 11.11). Let $X, Y \in$ $L^{\infty}$. It holds for $i=0, \ldots, N$,

R1: Cash invariance: $p_{i}\left(X+X_{i}\right)=p_{i}(X)+X_{i}$ for any $X_{i} \in L_{i}^{\infty}$.

R2: Monotonicity: $X \leq Y \Rightarrow p_{i}(X) \leq p_{i}(Y)$.

R3: Sub-additivity: $p_{i}(X+Y) \leq p_{i}(X)+p_{i}(Y)$.

R4: Positive homogeneity: $p_{i}(\lambda X)=\lambda p_{i}(X)$, where $\lambda \in L_{i}^{\infty}$ and $0 \leq \lambda$.

R5: Continuity from below: It holds $X_{n} \nearrow X \Rightarrow p_{i}\left(X_{n}\right) \nearrow p_{i}(X)$ for any sequence $\left(X_{n}\right) \subset L^{\infty}$.

R6: Time-consistency: $p_{i}(X)=p_{i}\left(p_{i+1}(X)\right), i=0, \ldots, N-1$.

We additionally assume

R7: No-arbitrage: $E_{\mathbb{Q}}\left(X \mid \mathcal{F}_{i}\right) \leq p_{i}(X)$. 
Jouini (2000) modelled the risk-free bank account as perfectly liquid. Property R1 states the same: investors may insert or withdraw any amount of cash to or from the risk-free bank account without transaction costs. Properties R2-R5 have been proposed in similar form and are discussed by Jouini and Kallal (1995, 2001) and Jouini (2000). Time-consistency has been introduced by Peng (2004) for nonlinear expectations. It means that prices behave consistently over time: prices can be computed either directly or using an intermediate instant of time, see Bion-Nadal (2009). Property R7 guarantees that the bid-ask spread is always greater or equal to zero and that the market is arbitrage-free, see Proposition 2.1. Our model of bid and ask prices can be seen as a discrete version of the continuous time model via dynamic convex risk-measures developed by BionNadal (2009).

Bid and ask prices of a contingent claim $C \in L^{\infty}$ at trading period $i$ are defined by

$$
\operatorname{bid}_{i}(C):=-p_{i}(-C) \text { and } \operatorname{ask}_{i}(C):=p_{i}(C), \quad i=0, \ldots, N,
$$

i.e. as in Jouini and Kallal (1995), Staum (2004) and Bion-Nadal (2009), we consider that selling $C$ is the same as buying $-C$. By property R1, we assume that at the end of the time-horizon the bid-ask spread of $C$ is zero. We therefore do not have to distinguish between contingent claims with asset delivery and cash settlement. Bid and ask prices of the risky asset are then defined by the processes

$$
S_{i}^{b}:=-p_{i}\left(-S_{N}\right) \text { and } S_{i}^{a}:=p_{i}\left(S_{N}\right), \quad i=0, \ldots, N .
$$

American contingent claims can be described by adapted stochastic processes, bid and ask prices of such claims are defined in Section 2.3.

We call the tuple $\left(\left(B_{i}\right),\left(S_{i}^{b}\right),\left(S_{i}^{a}\right),\left(p_{i}\right)\right)$ a security price model. We show that our security price model does not admit arbitrage. Furthermore, it is not possible to construct a self-financing portfolio, which super-replicates $C$ but can be bought for less than $p_{0}(C)$. The proof can be found in the appendix.

Proposition 2.1. The security price model $\left(\left(B_{i}\right),\left(S_{i}^{b}\right),\left(S_{i}^{a}\right),\left(p_{i}\right)\right)$ admits no arbitrage and the ask price $p_{0}(C)$ of a contingent claim $C \in L^{\infty}$ is less or equal to the price of the smallest self-financing trading strategy dominating $C$.

\subsection{Concave Distortion Functions}

In this Section, we introduce a parametric model for the pricing functional $\left(p_{i}\right)$. We will allow the pricing functional to depend on a parameter $\gamma \geq 0$ with the following interpretation: the greater $\gamma$, the greater the bid-ask spread, for $\gamma=0$, the spread is equal to zero. To obtain such parametrization, we let the coherent risk measures, defining the pricing functional, be based on a family of concave distortion functions, which is defined as follows:

Definition 2.2. A family of concave distortion functions (FCDF) $\left(\Psi^{\gamma}\right)_{\gamma \geq 0}$ is a set of functions $\Psi^{\gamma}:[0,1] \rightarrow[0,1]$ that are monotonically increasing, continuous and concave for all $\gamma \geq 0$ and for which $\Psi^{\gamma}(0)=0$ and $\Psi^{\gamma}(1)=1$. Moreover 
the family is monotonically increasing and continuous at $\gamma$, i.e. it holds that $\Psi^{\gamma_{1}}(u) \leq \Psi^{\gamma_{2}}(u)$ for $\gamma_{1} \leq \gamma_{2}$ and the map $\gamma \mapsto \Psi^{\gamma}(u)$ is continuous for all $u \in[0,1]$.

Additional, we make the following two assumption:

A1 For $\gamma=0$, the FCDF is equal to the identity, i.e.

$$
\Psi^{0}(u)=u, \quad u \in[0,1]
$$

A2 The total differential of the $\operatorname{FCDF}$ exists at the point $(u, \gamma)=\left(\frac{1}{2}, 0\right)$, i.e.

$$
\Psi^{\xi_{\gamma}}\left(\frac{1}{2}+\xi_{p}\right)=\frac{1}{2}+\xi_{u}+\frac{1}{2} \xi_{\gamma}+o\left(\left|\xi_{u}\right|+\left|\xi_{\gamma}\right|\right), \quad \xi_{u} \in(0,1), \quad \xi_{\gamma}>0 .
$$

Equation (1) holds, if the function $(u, \gamma) \mapsto \Psi^{\gamma}(u)$ is partial differentiable and all partial derivatives at the point $\left(\frac{1}{2}, 0\right)$ are continuous with

$$
\left.\frac{\partial}{\partial u} \Psi^{\gamma}(u)\right|_{(u, \gamma)=\left(\frac{1}{2}, 0\right)}=1 \text { and }\left.\frac{\partial}{\partial \gamma} \Psi^{\gamma}(u)\right|_{(u, \gamma)=\left(\frac{1}{2}, 0\right)}=\frac{1}{2} .
$$

Assumption A2 is used to prove convergence of bid and ask prices. By Assumption A1 the FCDF $\left(\Psi^{\gamma}\right)$ fulfils $\Psi^{0}\left(\frac{1}{2}\right)=\frac{1}{2}$. All FCDF satisfying Assumption A2 are also (approximately) equal in a small neighbourhood around $(u, \gamma)=\left(\frac{1}{2}, 0\right)$. Therefore we will see that the particular choice of the FCDF to model the pricing functional in the discrete time model does not matter when the number of trading periods tend to infinity.

Example 2.3. The family of distortion function corresponding to the expected shortfall can be defined by

$$
\Psi_{\text {ExpShortfall }}^{\gamma}(u)=\min (u(1+\gamma), 1), \quad u \in[0,1], \quad \gamma \geq 0
$$

The FCDF corresponding to the expected shortfall satisfies Equation (2) because for $u$ close enough to $\frac{1}{2}$ and $\gamma$ close enough to zero, it holds

$$
\Psi_{\text {ExpShortfall }}^{\gamma}(u)=u+u \gamma \text {. }
$$

Example 2.4. Let $\Phi$ be the cumulative standard normal distribution function and $\varphi$ the normal density. The WANG-transform

$$
\Psi_{\mathrm{WANG}}^{\gamma}(u)=\Phi\left(\Phi^{-1}(u)+\frac{1}{2 \varphi(0)} \gamma\right), u \in[0,1], \quad \gamma \geq 0
$$

was introduced by Wang (2000) and fulfils Equation (2). The WANG-transform is widely used in actuarial science and was originally defined without the scaling factor $\frac{1}{2 \varphi(0)}$.

Many other FCDF known in literature fulfil Assumptions A1 and A2, in particular the FCDF MINVAR, MAXVAR, MINMAXVAR and MAXMINVAR introduced by Cherny and Madan (2009). 


\subsection{Coherent Risk measures induced by Concave Distor- tion Functions}

Given a $\operatorname{FCDF}\left(\Psi^{\gamma}\right)$, the map

$$
\rho^{\gamma}(X):=\int_{0}^{\infty}\left(\Psi^{\gamma}(\mathbb{Q}[X<y])-1\right) d y+\int_{-\infty}^{0} \Psi^{\gamma}(\mathbb{Q}[X<y]) d y, \quad \gamma \geq 0
$$

defines a static coherent risk measure in the sense of Artzner et al. (1999), see Föllmer and Schied (2011, Theorem 4.70). The greater $\gamma$, the more conservative the risk measure. As in Madan, Pistorius and Schoutens (2012, 2017) and Leippold and Schärer (2017), we generalize this risk measure to the dynamic case. For $i=0, \ldots, N$ let

$$
\tilde{\rho}_{i}^{\gamma}(X):=\int_{0}^{\infty}\left(\Psi^{\gamma}\left(\mathbb{Q}_{i}[X<y]\right)-1\right) d y+\int_{-\infty}^{0} \Psi^{\gamma}\left(\mathbb{Q}_{i}[X<y]\right) d y, \quad \gamma \geq 0,
$$

where

$$
\mathbb{Q}_{i}(A):=E_{\mathbb{Q}}\left(1_{A} \mid \mathcal{F}_{i}\right), \quad i=0, \ldots, N,, A \in \mathcal{F},
$$

is a conditional probability. Define

$$
\rho_{N}^{\gamma}:=\tilde{\rho}_{N}^{\gamma}
$$

and recursively

$$
\rho_{i}^{\gamma}:=\tilde{\rho}_{i}^{\gamma}\left(-\rho_{i+1}^{\gamma}\right), \quad i=0, \ldots, N-1, \quad \gamma \geq 0 .
$$

The pricing functional used in this article is then defined by

$$
p_{i}^{\gamma}(.):=\rho_{i}^{\gamma}(-.), \quad i=0, \ldots, N, \gamma \geq 0 .
$$

The recursive definition makes the pricing functional time-consistent. By assumption A1 it holds

$$
E_{\mathbb{Q}}\left[X \mid \mathcal{F}_{i}\right]=p_{i}^{0}(X) .
$$

The parameter $\gamma \geq 0$ describes the liquidity of the market: the greater $\gamma$, the greater the bid-ask spread. For $\gamma=0$, bid and ask prices coincide and are identical to the risk neutral price operator.

Due to the time-consistency, for a fixed $\gamma_{N} \geq 0$, bid and ask prices of a future random cash flow $C^{E} \in L^{\infty}$ can be obtained by recursions:

$$
\begin{aligned}
\operatorname{bid}_{N}\left(C^{E}\right) & =\operatorname{ask}_{N}\left(C^{E}\right)=C^{E}, \\
\operatorname{bid}_{i}\left(C^{E}\right) & =-p_{i}^{\gamma_{N}}\left(-\operatorname{bid}_{i+1}\left(C^{E}\right)\right), \quad i=0, . ., N-1, \\
\text { and } \operatorname{ask}_{i}\left(C^{E}\right) & =p_{i}^{\gamma_{N}}\left(\operatorname{ask}_{i+1}\left(C^{E}\right)\right), \quad i=0, . ., N-1 .
\end{aligned}
$$

We explicitly allow the parameter $\gamma_{N}$, which describes the bid-ask spread in the $N^{\text {th }}$ model, to depend on $N$, in order to obtain convergence results for $N \rightarrow \infty$. 


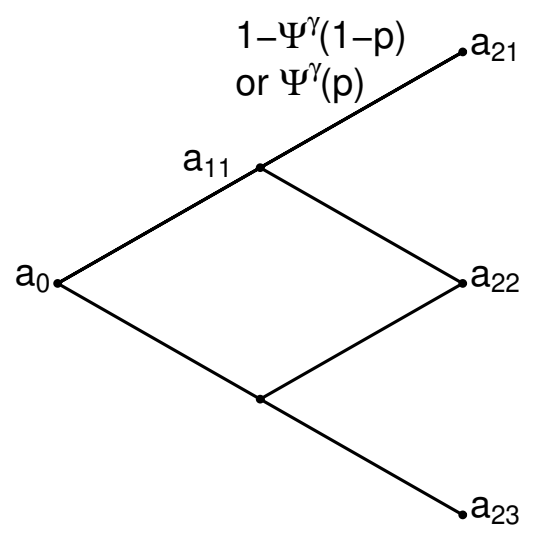

Figure 1: Binomial Tree to compute the ask price. The up-move probability depends on the sorting of the successive nodes.

Remark 2.5. The bid and ask prices are recursively defined and can be computed going backwards through a tree. Figure 1 shows a binomial tree with $N=2$ time-steps. The ask prices at the final nodes are equal to the value of the option at expiration. The ask price at the first node (today) can be computed going iteratively through the tree using the recursions (5). For example the ask price $a_{11}$ can be computed using the two successive nodes $a_{21}$ and $a_{22}$.

$$
a_{11}= \begin{cases}\Psi^{\gamma}(1-p) a_{22}+\left(1-\Psi^{\gamma}(1-p)\right) a_{21} & , a_{21} \leq a_{22} \\ \left(1-\Psi^{\gamma}(p)\right) a_{22}+\Psi^{\gamma}(p) a_{21} & , a_{21}>a_{22},\end{cases}
$$

where $p$ denotes the up-move probability in a classical binomial model and $\left(\Psi^{\gamma}\right)$ is a FCDF. The formula is deduced from the definition of pricing functional, see Equation (4). In contrast to the iterative computation of the risk-neutral price in the classical binomial model, the bid and ask prices depend on the sorting of the successive nodes. Therefore in this article we only prove convergence for monotone payoffs, which are precisely defined in Section 2.3. Bid and ask prices of general payoffs can be computed in the discrete time model going backwards through the tree and checking at each node the sorting of the two successive nodes.

\subsection{Payoffs}

The main goal of this article is to prove convergence of bid and ask prices of different European and American contingent claims, when the number of trading periods approaches infinity. We focus on contingent claims which are monotonically increasing or decreasing with respect to the underlying, this has a technical reason, see Remark 2.5. In the following we precisely define increasing and decreasing European and American contingent claims and provide a selection of examples. 
Definition 2.6. An European contingent claim $C^{E}$ is a bounded random variable on $(\Omega, \mathcal{F})$, such that there is a measurable function $h$, with

$$
C^{E}=h\left(S_{0}, \ldots, S_{N}\right) .
$$

The claim is called increasing if

$$
h\left(x_{0}, \ldots, x_{N}\right) \geq h\left(y_{0}, \ldots, y_{N}\right), x_{i} \geq y_{i}, \quad i=0, \ldots, N
$$

and decreasing if

$$
h\left(x_{0}, \ldots, x_{N}\right) \leq h\left(y_{0}, \ldots, y_{N}\right), \quad x_{i} \geq y_{i}, \quad i=0, \ldots, N .
$$

An American contingent claim $C^{A}$ is a bounded adapted process

$$
C^{A}=\left(C_{i}^{A}\right)_{i=0, . ., N},
$$

such that for each $i$ there is a measurable function $h_{i}$, with

$$
C_{i}^{A}=h_{i}\left(S_{0}, \ldots, S_{i}\right) .
$$

The claim is called increasing if

$$
h_{i}\left(x_{0}, \ldots, x_{i}\right) \geq h_{i}\left(y_{0}, \ldots, y_{i}\right), x_{k} \geq y_{k}, i=0, \ldots, N, \quad, k=0, \ldots, i
$$

and decreasing if

$$
h_{i}\left(x_{0}, \ldots, x_{i}\right) \leq h_{i}\left(y_{0}, \ldots, y_{i}\right), x_{k} \geq y_{k}, i=0, \ldots, N, \quad, k=0, \ldots, i .
$$

A European claim $C^{E}$ can be interpreted as a random payoff at maturity $T$. For each $i$, the random variable $C_{i}^{A}$ is interpreted as the payoff of the American contingent claim if the claim is exercised after $i$ trading periods. We assume the American option is cash-settled, and the reference price is the process $\left(S_{i}\right)$. If the holder of an American option exercises the option early after $i$ trading periods, she will receive the amount $h_{i}\left(S_{0}, \ldots, S_{i}\right)$, which is independent of the current bid-ask spread or the processes $\left(S_{i}^{b}\right)$ and $\left(S_{i}^{a}\right)$. This may in particular hold for cash-settled index options and it holds approximately for options with physically delivery if the transaction costs of trading the stock are small. Similar to European contingent claims, see Equation (5), bid and ask prices of an American contingent claim $C^{A}$ can be defined recursively, incorporating the possibility of an early exercise:

$$
\begin{aligned}
\operatorname{bid}_{N}\left(C^{A}\right) & =\operatorname{ask}_{N}\left(C^{A}\right)=C_{N}^{A}, \\
\operatorname{bid}_{i}\left(C^{A}\right) & =C_{i}^{A} \vee-p_{i}^{\gamma_{N}}\left(-\operatorname{bid}_{i+1}\left(C^{A}\right)\right), \quad i=0, . ., N-1, \\
\text { and } \operatorname{ask}_{i}\left(C^{A}\right) & =C_{i}^{A} \vee p_{i}^{\gamma_{N}}\left(\operatorname{ask}_{i+1}\left(C^{A}\right)\right), \quad i=0, . ., N-1 .
\end{aligned}
$$

Let $\Xi$ be the set all American contingent claims. To simplify notation, the operators assigning bid and ask prices to American contingent claims

$$
\operatorname{bid}_{i}: \Xi \rightarrow L_{i}^{\infty} \text { and } \operatorname{ask}_{i}: \Xi \rightarrow L_{i}^{\infty},
$$


have the same names as the operators describing prices of European contingent claims, which can be seen as functionals from $L^{\infty}$ to $L_{i}^{\infty}$.

We provide some examples of European and American contingent claims. Let $K \geq 0$ be a strike price and $B \geq 0$ be a barrier. By $\mathcal{N}$ we denote the time, the option is exercised. If $\mathcal{N} \in\{0, \ldots, N\}$ can be chosen by the holder of the option, we speak of an American contingent claim, exercised at time $\mathcal{N}$. If only $\mathcal{N}=N$ is allowed, i.e. the option can only be exercised at maturity, we speak of an European contingent claim.

Example 2.7. The following derivatives are increasing contingent claims.

- Call option: $C_{\text {Call }}=\left(S_{\mathcal{N}}-K\right)^{+}$

- Lookback call option: $C_{\text {LbCall }}=\left(\max _{i \in\{0, \ldots, \mathcal{N}\}} S_{i}-K\right)^{+}$

- Asian call option: $C_{\text {AsianCall }}=\left(\frac{1}{\mathcal{N}} \sum_{i=0}^{\mathcal{N}} S_{i}-K\right)^{+}$

- Barrier up-and-in call option:

$$
C_{\text {UICall }}= \begin{cases}\left(S_{\mathcal{N}}-K\right)^{+} & \max _{i \in\{0, \ldots, \mathcal{N}\}} S_{i} \geq B \\ 0, & \text { otherwise }\end{cases}
$$

- Barrier down-and-out call option:

$$
C_{\text {DOCall }}= \begin{cases}\left(S_{\mathcal{N}}-K\right)^{+} & \min _{i \in\{0, \ldots, \mathcal{N}\}} S_{i}>B \\ 0, & \text { otherwise }\end{cases}
$$

Example 2.8. Decreasing payoffs are for example:

- Put option: $C_{\text {Call }}=\left(K-S_{\mathcal{N}}\right)^{+}$

- Lookback put option: $C_{\mathrm{LbPut}}=\left(K-\max _{i \in\{0, \ldots, \mathcal{N}\}} S_{i}\right)^{+}$

- Asian put option: $C_{\mathrm{AsianPut}}=\left(K-\frac{1}{\mathcal{N}} \sum_{i=0}^{\mathcal{N}} S_{i}\right)^{+}$

- Barrier up-and-out put option:

$$
C_{\text {UOPut }}= \begin{cases}\left(K-S_{\mathcal{N}}\right)^{+} & , \max _{i \in\{0, \ldots, \mathcal{N}\}} S_{i}<B \\ 0, & \text { otherwise. }\end{cases}
$$

Example 2.9. The following two derivatives are neither increasing nor decreasing payoffs. 
- Barrier up-and-out call option

$$
C_{\text {UOCall }}= \begin{cases}\left(S_{\mathcal{N}}-K\right)^{+} & , \max _{i \in\{0, \ldots, \mathcal{N}\}} S_{i}<B \\ 0, & \text { otherwise }\end{cases}
$$

- Barrier down-and-in call option

$$
C_{\text {DICall }}= \begin{cases}\left(S_{\mathcal{N}}-K\right)^{+} & , \min _{i \in\{0, \ldots, \mathcal{N}\}} S_{i} \leq B \\ 0, & \text { otherwise. }\end{cases}
$$

\section{Classical Binomial Model}

In this Section, we recall the classical binomial model. Let $T>0$ be some time-horizon and assume there are $N \in \mathbb{N}$ trading periods between $[0, T]$, each trading period is of length $\frac{T}{N}$. There is a riskless bond

$$
B_{i}^{(N)}=\left(1+r_{N}\right)^{i}, \quad i=0,1, \ldots, N
$$

paying interest $r_{N}=\frac{r T}{N}>-1$ in each trading period and just one risky asset, paying dividends $q_{N}=\frac{q T}{N}$ in each period, and whose price process takes the form

$$
S_{i}^{(N)}=S_{0} \prod_{k=1}^{i}\left(1+R_{k}^{(N)}\right), \quad i=1,2, \ldots, N,
$$

where $S_{0}>0$, and the returns

$$
R_{i}^{(N)}=\frac{S_{i}^{(N)}-S_{i-1}^{(N)}}{S_{i-1}^{(N)}}, i=1,2, \ldots, N
$$

are random variables with values in $\left\{a_{N}, b_{N}\right\} \subset \mathbb{R}$, such that

$$
d_{N}:=1+a_{N}=e^{-\sigma \sqrt{\frac{T}{N}}} \text { and } u_{N}:=1+b_{N}=e^{\sigma \sqrt{\frac{T}{N}}} .
$$

The market is arbitrage-free and complete if $-1<a_{N}<r_{N}-q_{N}<b_{N}$, which holds for $N$ large enough. In this case, the returns $R_{1}^{(N)}, \ldots, R_{N}^{(N)}$ are independent, their distributions are characterized by

$$
P_{N}^{*}\left(R_{i}^{(N)}=b_{N}\right):=p_{N}^{*}:=p_{N}^{q}+\varphi(N), i=1,2, \ldots, N,
$$

where $P_{N}^{*}$ is the unique risk neutral measure, $\varphi(N) \in o\left(\frac{1}{\sqrt{N}}\right)$ and

$$
p_{N}^{q}:=\frac{1}{2}+\frac{r-q}{2 \sigma} \sqrt{\frac{T}{N}} .
$$

We say the classical binomial model is characterized by the tuple $\left(S_{0}, r, q, \sigma, T, N\right)$. 
Let $C_{E}^{(N)}$ be a possibly path-dependent European contingent claim. The discounted claim

$$
H_{E}^{(N)}=\frac{C_{E}^{(N)}}{B_{N}^{(N)}}
$$

can be written as

$$
H_{E}^{(N)}=h\left(S_{0}^{(N)}, \ldots, S_{N}^{(N)}\right)
$$

for a suitable function $h$. The value process in the $N^{\text {th }}$ model,

$$
V_{i}^{(N)}=E_{P_{N}^{*}}\left[H_{E}^{(N)} \mid \mathcal{F}_{i}\right], i=1,2, \ldots, N,
$$

of a replicating strategy for $H_{E}^{(N)}$ at time $t=\frac{i T}{N}$ is of the form

$$
V_{i}^{(N)}(\omega)=v_{i}^{(N)}\left(S_{0}, S_{1}(\omega), . ., S_{i}(\omega)\right)
$$

where the function $v_{i}^{(N)}$ is given by recursion

$$
\begin{aligned}
v_{N}^{(N)}\left(x_{0}, \ldots, x_{N}\right)= & h\left(x_{0}, \ldots, x_{N}\right) \\
v_{i}^{(N)}\left(x_{0}, \ldots, x_{i}\right)= & \left(1-p_{N}^{*}\right) v_{i+1}^{(N)}\left(x_{0}, \ldots, x_{i}, x_{i} d_{N}\right) \\
& +p_{N}^{*} v_{i+1}^{(N)}\left(x_{0}, \ldots, x_{i}, x_{i} u_{N}\right), \quad i=0,1, \ldots, N-1,
\end{aligned}
$$

see e.g. Föllmer and Schied (2011, Proposition 5.41).

On the other hand, dealing with an American contingent claim

$$
C_{A}^{(N)}=\left(C_{A, i}^{(N)}\right)_{i=0, . ., N},
$$

and the corresponding discounted claim

$$
H_{A, i}^{(N)}=\frac{C_{A, i}^{(N)}}{B_{i}^{(N)}}, \quad i=0, . ., N,
$$

for each $i=0,1, . ., N$ there is a suitable function $h_{i}$ such that

$$
H_{A, i}^{(N)}=h_{i}\left(S_{0}, \ldots, S_{i}\right) .
$$

By no-arbitrage-arguments, the value process $\left(V_{i}\right)_{i=0, \ldots, N}$ of a replicating strategy for $H_{A}^{(N)}$ can be found by recursion, compare with Föllmer and Schied (2011, Chapter 6):

$$
V_{N}:=H_{A, N}^{(N)}, \quad V_{i}:=H_{A, i}^{(N)} \vee E_{P_{N}^{*}}\left[V_{i+1} \mid \mathcal{F}_{i}\right], \quad i=0, . ., N-1 .
$$

Hence, there are functions $v_{i}^{(N)}$ such that

$$
V_{i}=v_{i}^{(N)}\left(S_{0}, \ldots, S_{i}\right), \quad i=0, \ldots, N,
$$


namely

$$
\begin{aligned}
v_{N}^{(N)}\left(x_{0}, \ldots, x_{N}\right)= & h_{N}\left(x_{0}, \ldots, x_{N}\right) \\
v_{i}^{(N)}\left(x_{0}, \ldots, x_{i}\right)= & h_{i}\left(x_{0}, \ldots, x_{i}\right) \vee\left\{\left(1-p_{N}^{*}\right) v_{i+1}^{(N)}\left(x_{0}, \ldots, x_{i}, x_{i} d_{N}\right)\right. \\
& \left.+p_{N}^{*} v_{i+1}^{(N)}\left(x_{0}, \ldots, x_{i}, x_{i} u_{N}\right)\right\}, i=0,1, \ldots, N-1 .
\end{aligned}
$$

It is well known that in a classical binomial-tree model, which is characterized by the tuple $\left(S_{0}, r, q, \sigma, T, N\right)$, the risk-neutral price

$$
\pi^{(N)}\left(C^{(N)}, S_{0}, r, q, \sigma, T\right):=v_{0}^{(N)}\left(S_{0}\right)
$$

of an European or American contingent claim $C^{(N)}=C_{E}^{(N)}$ or $C^{(N)}=C_{A}^{(N)}$, converge for many products as $N \rightarrow \infty$. If the limit exists, we define

$$
\pi\left(C, S_{0}, r, q, \sigma, T\right):=\lim _{N \rightarrow \infty} \pi^{(N)}\left(C^{(N)}, S_{0}, r, q, \sigma, T\right) .
$$

Convergence of plain vanilla European options to the Black-Scholes price are discussed in Cox, Ross and Rubinstein (1979). For plain vanilla American options we refer to Amin and Khanna (1994), for European and American Asian options and lookback options and some other path-dependent options, see Jiang and Dai (2004). For a proof of convergence for European barrier option, see Carbone (2004) and Lin and Palmer (2013) and references therein. Those convergence results can directly be applied to prove convergence of bid and ask prices as Theorem 4.1 shows.

\section{Convergence of Bid and Ask Prices}

In this Section we prove our main result and show that bid and ask prices of European or American contingent claims converge, if the risk-neutral price of the claim converges in the classical binomial model. The theorem has an important practical implication: is states that bid and ask prices of monotone payoffs, in particular plain vanilla European and American options, can be computed using the classical Black-Scholes model with an adjusted drift. Bid and ask prices of such options can therefore be computed very fast.

Theorem 4.1. Let $\left(\Psi^{\gamma}\right)_{\gamma>0}$ be a FCDF fulfilling Assumption A1 and A2. Let a classical binomial-tree model be given, which is characterized by the tuple

$$
\left(S_{0}, r, q, \sigma, T, N\right) \text {. }
$$

Let $C^{(N)}$ be an increasing (decreasing) European or American contingent claim. Let $\gamma \geq 0$ and

$$
\gamma_{N}:=\gamma \sqrt{\frac{T}{N}} .
$$


Define bid and ask prices of an European claim by recursions (5) and of an American claim by recursions (6). If risk-neutral-price, defined via Equation (11),

$$
\pi^{(N)}\left(C^{(N)}, S_{0}, r, \tilde{q}, \sigma, T\right)
$$

converges in the classical binomial model for all dividends

$$
\tilde{q} \in[q-\sigma \gamma, q+\sigma \gamma]
$$

to some non-negative number $\pi\left(C, S_{0}, r, \tilde{q}, \sigma\right)$, then the ask (bid) of the contingent claim converges to

$$
\lim _{N \rightarrow \infty} \pi^{(N)}\left(C^{(N)}, S_{0}, r, q-\sigma \gamma, \sigma\right)
$$

and the bid (ask) price converges to

$$
\lim _{N \rightarrow \infty} \pi^{(N)}\left(C^{(N)}, S_{0}, r, q+\sigma \gamma, \sigma\right) .
$$

Proof. The proof can be found in the appendix.

A look at the proof of Theorem 4.1 shows that one could define $\gamma_{N}$ in Equation (12) arbitrarily, as long as it converges to zero as fast as $\frac{1}{\sqrt{N}}$. Under our particular choice, $\gamma$ can be interpreted as a drift-adjustment via the dividend yield in the continuous time limit scaled by the volatility. The drift adjustment is

$$
\hat{q} T=(q \pm \sigma \gamma) T .
$$

Remark 4.2. In the binomial model, the underlying is modelled by a bounded stochastic process. Therefore there is no restriction of the definition of bid and ask prices via recursions (5) and (6) requiring the contingent claims to be bounded. In particular call options are bounded in discrete time. Bid and ask prices of a contingent claim form a two-dimensional sequence with the natural numbers $1,2,3, .$. as index set. The index $N$ corresponds to the $N^{\text {th }}$ binomial model. In Theorem 4.1, we prove convergence of such a sequence. Hence Theorem 4.1 says that bid and ask prices of a possibly unbounded contingent claim like a European call option in continuous time can be approximated arbitrary closely by the bid and ask prices of a bounded contingent claim in discrete time.

\subsection{European Plain Vanilla Options}

In the classical Black-Scholes world, there exist closed-form solutions for the risk-neutral price of European plain vanilla and barrier options. By Theorem 4.1, we obtain closed-form solutions for bid and ask prices of European plain vanilla and barrier options, which are stated in the next corollaries, by taking the corresponding closed-form solutions for the risk-neutral price and adjusting the dividend yield. 
The Black-Scholes prices of plain vanilla European call and put options with strike $K$ and maturity $T$ are given in closed-form and denoted by

$$
\mathrm{BS}_{\text {Call }}\left(S_{0}, T, K, r, q\right)=S_{0} e^{-q T} \Phi\left(d_{1}\right)-e^{-r T} K \Phi\left(d_{2}\right)
$$

and

$$
\mathrm{BS}_{\mathrm{Put}}\left(S_{0}, T, K, r, q\right)=e^{-r T} K \Phi\left(-d_{2}\right)-S_{0} e^{-q T} \Phi\left(-d_{1}\right),
$$

where $d_{1}=\frac{\log \frac{S_{0}}{K}+\left(r-q+\frac{1}{2} \sigma^{2}\right) T}{\sigma \sqrt{T}}$ and $d_{2}=d_{1}-\sigma \sqrt{T}$ and $\Phi$ denotes the distribution function of the standard normal distribution, see Black and Scholes (1973).

Corollary 4.3. Under the notation of Theorem 4.1, let $C^{(N)}$ be an European plain vanilla option with strike $K>0$ and maturity $T$. Bid and ask prices of a put option converge to

$$
\operatorname{bid}_{\mathrm{Put}}^{\gamma}=\mathrm{BS}_{\mathrm{Put}}\left(S_{0}, T, K, r, q-\sigma \gamma\right)
$$

and

$$
\operatorname{ask}_{\mathrm{Put}}^{\gamma}=\mathrm{BS}_{\mathrm{Put}}\left(S_{0}, T, K, r, q+\sigma \gamma\right) .
$$

Bid and ask prices of a call option converge to

$$
\operatorname{bid}_{\text {Call }}^{\gamma}=\operatorname{BS}_{\text {Call }}\left(S_{0}, T, K, r, q+\sigma \gamma\right)
$$

and

$$
\operatorname{ask}_{\text {Call }}^{\gamma}=\operatorname{BS}_{\text {Call }}\left(S_{0}, T, K, r, q-\sigma \gamma\right) \text {. }
$$

Figure 2 shows the relative bid-ask spread $\frac{\text { ask-bid }}{(\text { ask+bid }) \frac{1}{2}}$ surface of European call options over strikes and maturities. Long term options and options being deep out-of-the money are less liquid, the relative bid-ask spread is greater.

Remark 4.4. Similarly to the existence of an implied volatility smile, there exist an implied liquidity smile. Computing $\gamma$ implicitly from given bid and ask prices of options, Corcuera et al. (2012) show that there is a non-linear dependence of $\gamma$, with respect to the term structure and the moneyness of the option surface. In particular, we cannot expect to predict the bid-ask spread of one option from given bid and ask prices of another option, if the corresponding strikes and maturities are too distant from each other.

\subsection{Path-dependent and American Options}

In a classical Black-Scholes framework, there exist closed-form solution for many barrier options, see Rubinstein and Reiner (1991) and Cheng (2003). For example the arbitrage-free price of an up-and-in barrier call option with maturity 


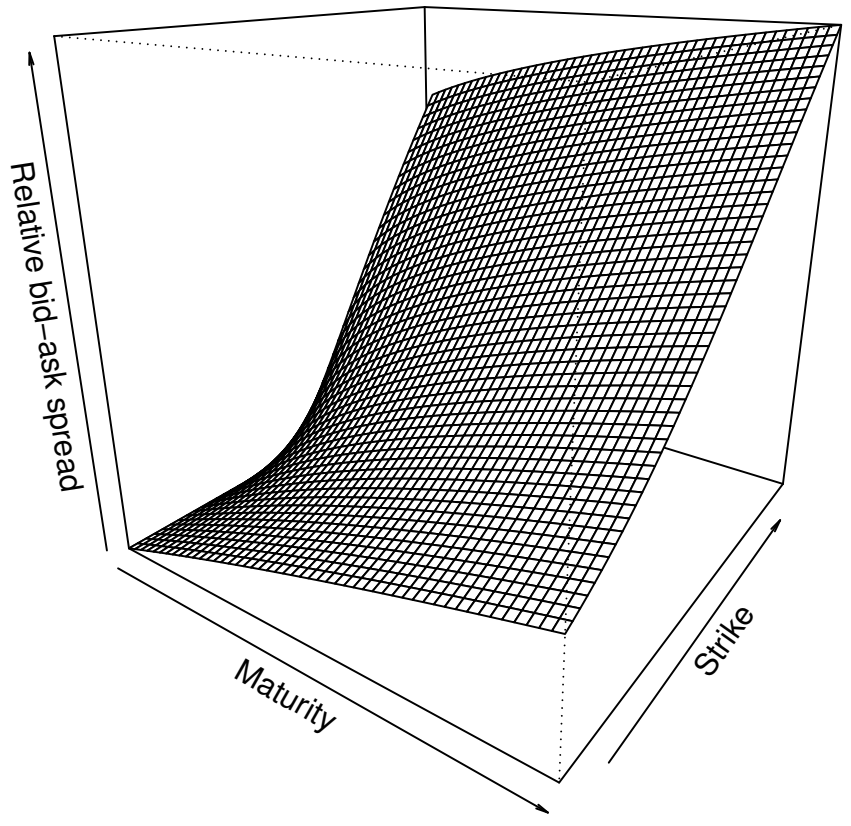

Figure 2: Relative bid-ask spread surface for European plain vanilla call options. We use the following parameters: underlying is equal to 100, the strikes vary between 50 and 150, annual interest rates are set to 0.01 , the dividend yield is assumed to be 0.03 , the time left to maturity lays in the interval $[0,2]$, the annual volatility is 0.2 and the annual $\gamma$ is set to 0.05 .

$T$, strike $K$ and barrier $B>K$ is

$$
\begin{aligned}
\mathrm{BS}_{\text {UICall }}\left(S_{0}, T, K, B, r, q\right)= & S_{0} e^{-q T} \Phi\left(x_{1}\right)-K e^{-r T} \Phi\left(x_{1}-\sigma \sqrt{T}\right) \\
& -S_{0} e^{-q T}\left(\frac{B}{S_{0}}\right)^{2 m}\left(\Phi(-y)-\Phi\left(-y_{1}\right)\right) \\
& +K e^{-r T}\left(\frac{B}{S_{0}}\right)^{2 m-2}(\Phi(-y+\sigma \sqrt{T}) \\
& \left.-\Phi\left(-y_{1}+\sigma \sqrt{T}\right)\right),
\end{aligned}
$$

where

$$
\begin{array}{rr}
m=\frac{r-q+\frac{1}{2} \sigma^{2}}{\sigma^{2}}, & y=\frac{\log \left(\frac{B^{2}}{S_{0} K}\right)}{\sigma \sqrt{T}}+m \sigma \sqrt{T}, \\
x_{1}=\frac{\log \left(\frac{S_{0}}{B}\right)}{\sigma \sqrt{T}}+m \sigma \sqrt{T}, \quad y_{1}=\frac{\log \left(\frac{B}{S_{0}}\right)}{\sigma \sqrt{T}}+m \sigma \sqrt{T} .
\end{array}
$$


Corollary 4.5. Under the notation of Theorem 4.1, let $C^{(N)}$ be an up-and-in barrier call option with maturity $T$, strike $K>0$, and barrier $B>K$. The bid price converges to

$$
\operatorname{bid}_{\mathrm{UICall}}^{\gamma}=B S_{\mathrm{UICall}}\left(S_{0}, T, K, B, r, q+\sigma \gamma\right)
$$

and the ask converges to

$$
\operatorname{ask}_{\mathrm{UICall}}^{\gamma}=B S_{\mathrm{UICall}}\left(S_{0}, T, K, B, r, q-\sigma \gamma\right) .
$$

In the following, we treat bid prices of American put options but the findings can be transferred directly to ask prices of American put options and American call options as well. Let the risk-neutral Black-Scholes prices of a plain vanilla American put option with strike $K$ and maturity $T$ be denoted by

$$
\mathrm{BS}_{\mathrm{Put}}^{\mathrm{A}}\left(S_{0}, T, K, r, q\right) \text {. }
$$

There are no closed-form solutions for American plain vanilla options in a classical Black-Scholes framework, but there exist efficient numerical methods to approximate $\mathrm{BS}_{\mathrm{Put}}^{\mathrm{A}}$, see for example Barone-Adesi and Whaley (1987) and Bjerksund and Stensland (1993). We denote the numerical approximation by

$$
\widetilde{\mathrm{BS}}_{\mathrm{Put}}^{\mathrm{A}}\left(S_{0}, T, K, r, q\right)
$$

and the error by

$$
\varepsilon_{\mathrm{Put}}^{q}:=\left|\mathrm{BS}_{\mathrm{Put}}^{\mathrm{A}}\left(S_{0}, T, K, r, q\right)-\widetilde{\mathrm{BS}}_{\mathrm{Put}}^{\mathrm{A}}\left(S_{0}, T, K, r, q\right)\right| .
$$

The next corollary follows immediately:

Corollary 4.6. Under the notation of Theorem 4.1, let $C^{(N)}$ be an American plain vanilla put option with strike $K>0$ and maturity $T$. The bid price converge to

$$
\operatorname{bid}_{\mathrm{Put}}^{\gamma}=\mathrm{BS}_{\mathrm{Put}}^{\mathrm{A}}\left(S_{0}, T, K, r, q-\sigma \gamma\right) .
$$

The error approximating the bid price using $\widetilde{B S}_{P u t}^{A}$ as an estimate for $B S_{P u t}^{A}$ is less or equal to $\varepsilon_{\text {Put }}^{q-\sigma \gamma}$.

The corollary states the following: the bid price of an American put option on a stock with dividend yield $q$ is equal to the risk-neutral price of an American put option but on a stock with dividend yield $q-\sigma \gamma$. The bid price directly inherits the numerical error from the approximation of the the risk-neutral price of the American option by some numeric algorithm. A similar corollary could easily be stated for other options, like Asian options, which do not have closed-form solutions in the classical Black-Scholes model and can only be approximated for example with Monte Carlo methods. Bid and ask prices can then also be computed using Monte Carlo methods and the absolute error does not increase compared to classical risk-neutral pricing. 


\subsection{Numeric Simulations}

In this Section, we try to investigate how fast the recursively defined bid and ask prices converge. We make two approximations: we approximate the concave distortion function by a linear function, see Equation (1) and we approximate the Black-Scholes model by a binomial tree. The error of the first aproximation approaches zero faster than $\frac{1}{\sqrt{N}}$, see Equation (13).

The convergence rate of the classical binomial model is well studied in literature for many products: Heston and Zhou (2000) show that the risk-neutral price of a plain vanilla European call option converges at least as fast as $\frac{1}{\sqrt{N}}$. Lamberton (1998) prove that the risk-neutral price of an American put option converges from below and from above at least as fast as $N^{-\frac{2}{3}}$ and $N^{-\frac{3}{4}}$ respectively. Leisen and Reimer (1996) and Leisen (1998) analysed three different approaches to build a binomial tree, in particular the definitions for the returns of one trading period differ. They show that European plain vanilla options converge at least as fast as $\frac{1}{N}$ but American put options may only converges from below as fast as $\frac{1}{\sqrt{N}}$ depending on the exact tree definition. Lin and Palmer (2013) treat barrier options.

In our setting the up-move probability to obtain bid and ask prices has only asymptotically the martingale property, which makes it difficult to directly apply convergence results for classical binomial trees to our framework.

We therefore rely on simulations and compute bid and ask prices of an European call option, an American put option and an European up-and-in call option using recursions (5) and (6) for time-steps ranging from $N \in\{5, . ., 2000\}$. We compare the tree-prices to their continuous counterpart, which can be obtained via the Corollaries 4.3, 4.5 and 4.6. By

$$
e_{N}, \ldots, N \in \mathbb{N}
$$

we denote the absolute difference (error) between the bid or ask price of a contingent claim $C^{(N)}$ in the $N^{\text {th }}$ binomial model to the limiting bid or ask price. We say the sequence of errors converges with $\operatorname{order} \rho>0$, if there is a constant $\kappa>0$ such that

$$
\forall N \in \mathbb{N}: e_{N} \leq \frac{\kappa}{N^{\rho}} .
$$

The order of convergence can be indicated straightforwardly by a simulation, see Leisen and Reimer (1996). As

$$
\log \left(\frac{\kappa}{N^{\rho}}\right)=\log (\kappa)-\rho \log (N),
$$

the negative slope of a straight line obtained from a $\log -\log$ plot of the errors $e_{N}$ against the refinement $N$ can be used as an indicator for $\rho$. Figure 3 indicates an order of convergence between 1 and $\frac{1}{2}$ of the recursions (5) and (6) for different European and American options. 


\section{Convergence Rate for Several Options}

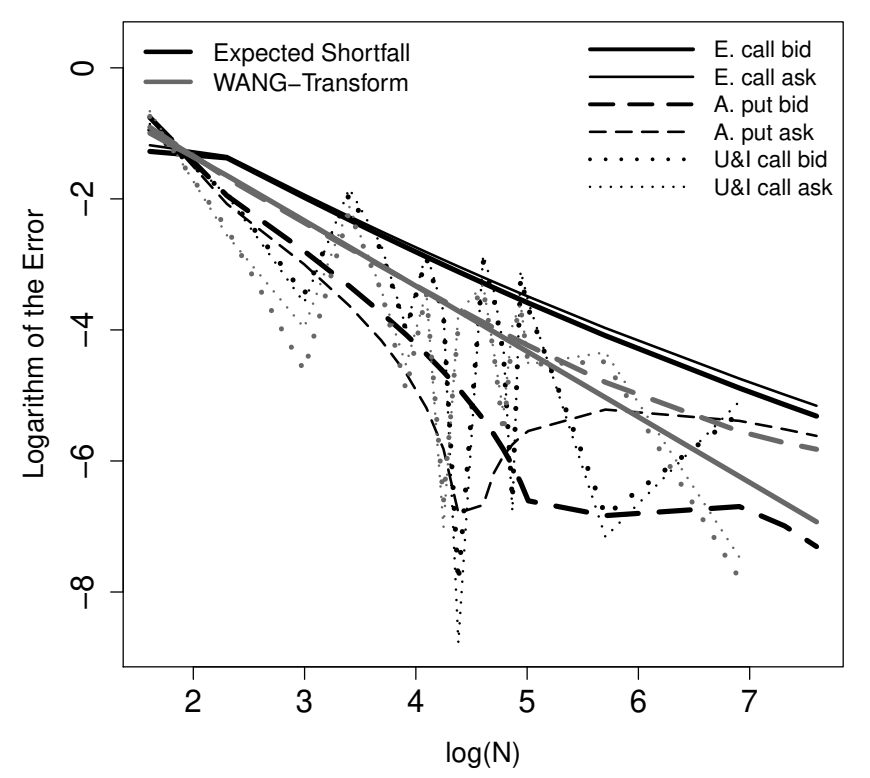

Figure 3: Log-log plot of the binomial tree approximation error for an European call option, an American put option and an up-and-in barrier option with barrier $B=110$. All option have the strike $K=100$ and the maturity is set to one year. The stock starts in $S_{0}=100$, annual interest rates are set to 0.01 , the dividend yield is assumed to be 0.03 , the annual volatility is 0.2 and the annual $\gamma$ is set to $0.05 . N$ goes in non-equidistant steps from 5 to 2000. The up-and-in barrier option is only simulated up to $N=1000$.

\section{$5 \quad$ Implied Liquidity (IL)}

In Guillaume et al. (2018), the concept of implied liquidity (IL) is defined, see also Corcuera et al. (2012), Dhaene et al. (2012) and Albrecher, Guillaume, and Schoutens (2013). It is similar to the idea of implied volatility and returns two implicitly computed parameters $\gamma_{b}$ and $\gamma_{a}$ such that modelled bid and ask prices match real market prices. The benefits of quoting the IL instead of bidask spreads are comparable to the benefits of quoting implied volatilities instead of mid-prices: in principle the IL can be constant across strikes, maturities and underlyings and hence makes it possible to compare bid-ask spreads across all three dimensions. Guillaume et al. (2018) computed the IL for European options and compared concept of the IL to the industrial standard of describing bid and ask price by quoting implied bid and ask volatilities. Their main finding is: a change in liquidity is described much more adequately by the IL than by the change of the difference of implied bid and ask volatilities. We repeat this 


\section{Change in Liquidity, ATM American Options}

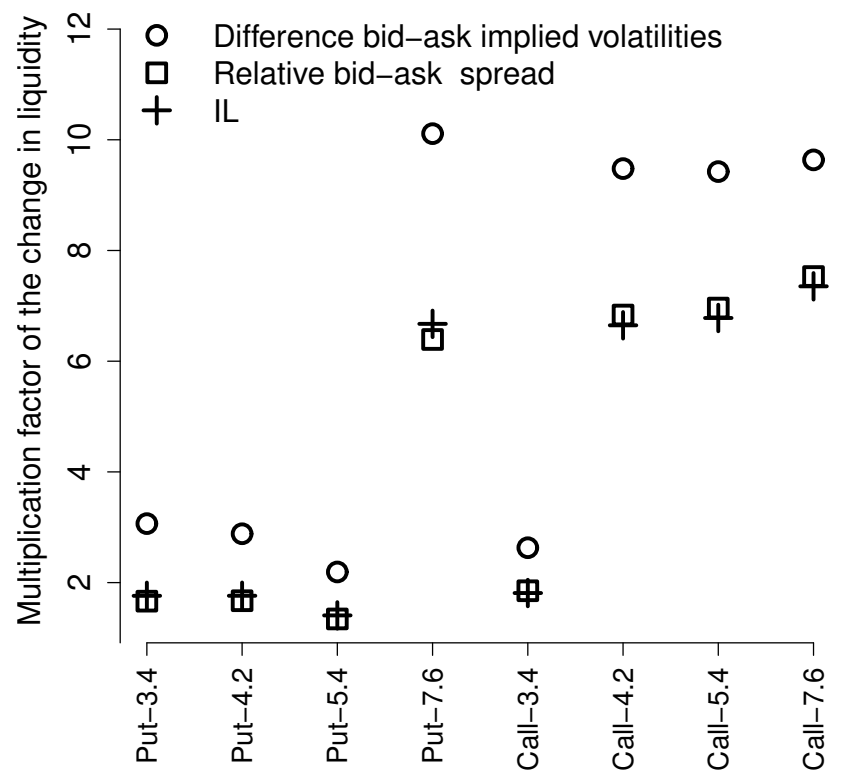

Figure 4: This Figure describes the change of the relative bid-ask spread, the IL and the difference of implied bid and ask volatilities from February, 2nd to February, 5th of various American at-the-money put and call options by a multiplicative factor. Maturities are measured in months.

empirical study for American options and obtain similar results.

For a time-series of two days, February, 2nd and February, 5th, 2018, we obtained end-of-day bid and ask prices of 80 plain vanilla, at-the-money American put and call options on the S\&P500, or rather the SPDR S\&P 500 ETF Trust, an exchange traded fund replicating the S\&P500, with maturities ranging from about 3 to 8 month. The option prices were obtained from the Chicago Board Options Exchange.

The CBOE Volatility Index (VIX), tracking short-term market volatility, jumped from 17.31 points on February, 2nd to 37.32 points on February, 5th, thus by $116 \%$, which is the highest daily relative change recorded so far. The S\&P500 lost about $4 \%$ between the two dates. It is well-known that liquidity dries up, when uncertainty in financial market rises. Therefore the chosen dates are well suited to analyse how different measures for the bid-ask spread behave, when liquidity changes.

For each American option, on both dates, we first compute an implied volatility $\sigma_{\text {Mid }}$ matching exactly the mid-price. Then we use the mid-price implied volatility and compute the IL, $\left(\gamma_{b}, \gamma_{a}\right) \in \mathbb{R}_{+}^{2}$, such that the model bid and ask prices match exactly the quoted market bid and ask prices. In particular for an 
American call option $C$, we solve numerically

$$
\operatorname{bid}_{\text {quoted market price }}(C)=\pi_{\text {Call }}^{A}\left(T, S_{0}, K, r, q+\sigma_{\mathrm{Mid}} \gamma_{b}, \sigma_{\mathrm{Mid}}\right),
$$

for $\gamma_{b}$. $\pi_{\text {Call }}^{A}\left(T, S_{0}, K, r, q, \sigma\right)$ is the risk-neutral price of an American call option in a Black-Scholes setting with strike $K$ and maturity $T$ on a stock with initial value $S_{0}$, volatility $\sigma$ and paying a continuously dividend yield $q$. The risk-free interest rate is denoted by $r$. The parameter $\gamma_{a}$ for the call option and the IL $\left(\tilde{\gamma}_{b}, \tilde{\gamma}_{a}\right)$ of put options can be found analogously, see Theorem 4.1. For most options $\gamma_{b}$ and $\gamma_{a}$ are almost identical.

The average relative bid-ask spread of the American option set is $1.6 \%$ on February, 2nd and $6.6 \%$ on February, 5th. The relative bid-ask spread changed by the factor 4.1. The average IL $\frac{\gamma_{a}+\gamma_{b}}{2}$, changed from an average value of 0.011 for all options on February, 2nd to 0.043 on February, 5th, which corresponds to a change by the factor 3.9. The average difference of implied bid and ask volatilities on the other hand, rose by the factor 6.1 , hence about $49 \%$ more than the relative bid-ask spread. In Figure 4, we show the multiplicative factor describing the change of the relative bid-ask spread, the IL and the difference of implied bid and ask volatilities from February, 2nd to February 5th separately for put and call options and different maturities.

The overall picture is the following: a change in liquidity of American options, due to a rise in uncertainty in the market and measured by the change of the relative bid-ask spread, is described by the IL very well. On the other hand, an overestimation of the change of liquidity by $50 \%$ and more are no exceptions, when describing the bid-ask spread by the classical way of quoting implied bid and ask volatilities. Our findings for American options are in line with a similar empirical study for European options done by Guillaume et al. (2018).

\section{Conclusion}

We model a financial market with frictions in discrete time by applying a pricing functional, which is defined recursively via coherent risk measures, to a classical binomial model. Economically, the discrete time model is justified in Jouini (2000). We are able to prove convergence of bid and ask prices for monotone products like European and American plain vanilla, Asian, lookback and (some) barrier options. In the limit we obtain an extended Black-Scholes formula containing a new parameter $\gamma \geq 0$, which adjusts the dividend yield. The greater $\gamma$, the greater the bid-ask spread. In particular for European plain vanilla options and barrier options exist closed-form solutions. The convergence results allow us to compute bid and ask prices of an option as fast as the risk-neutral price in a classical Black-Scholes model.

The new model may find a similar application in practise as the classical Black-Scholes model. Trader usually prefer to quote implied volatilities instead of prices, because there are many nonlinearities in prices making comparisons across strikes, maturities, and underlying assets difficult to comprehend and un- 
derstand. In principle volatilities could be constant across all three dimensions, hence the preference for quoting implied volatilities.

With the same argument it might be more convenient to quote an implicitly computed $\gamma$ instead of the bid-ask spread. To demonstrate this idea, we computed implicitly two parameters $\gamma_{b}$ and $\gamma_{a}$ for a set of American options such that modelled bid and ask prices match real market prices. In principle the tuple $\left(\gamma_{b}, \gamma_{b}\right)$ could be constant across strikes, maturities and underlyings and hence makes it possible to compare bid-ask spreads across all three dimensions.

We compare this new market implied liquidity measure to the common approach of describing the bid-ask spread by the difference between implied bid and ask Black-Scholes volatilities and show empirical that our model describes liquidity of American options over time significantly better. A similar study has been done by Guillaume et al. (2018) for European options using a static model.

A future research could consist of generalizing the binomial model and allowing in particular the parameter $\gamma$ and the volatility to be possibly correlated stochastic processes.

\section{Acknowledgements}

We thank two anonymous referees for many valuable comments and suggestions enabling us to greatly improve the quality of the paper.

\section{Appendix}

\section{Proof of Proposition 2.1.}

We trivially have

$$
0 \leq S_{i}^{b} \leq E_{\mathbb{Q}}\left(S_{N} \mid \mathcal{F}_{i}\right)=S_{i} \leq S_{i}^{a}, \quad i=0, \ldots, N,
$$

hence by Jouini and Kallal (1995, Theorem 3.2), the security price model admits no multiperiod free lunch and is hence arbitrage-free. Let

$$
\mathcal{A}_{0}:=\left\{X \in L^{\infty}, \rho_{0}(X) \leq 0\right\} .
$$

For a probability measure $Q$ equivalent to $\mathbb{Q}$, define

$$
\alpha_{0}^{\min }(Q):=\sup _{X \in \mathcal{A}_{0}} E_{Q}[X]
$$

It holds $\alpha_{0}^{\min }(\mathbb{Q}) \leq 0$, hence by Föllmer and Penner (2006, Corollary 4.12.), there exist a set of probability measures $\left(\mathcal{Q}^{e}\right)$, such that each element of $\mathcal{Q}^{e}$ is equivalent to $\mathbb{Q}$ and

$$
p_{i}(X)=\sup _{Q \in \mathcal{Q}^{e}} E_{Q}\left[X \mid \mathcal{F}_{i}\right], \quad i=0, . ., N .
$$


Let $\mathcal{P}$ be a set of probability measures containing all probability measures $P$ which are equivalent to $\mathbb{Q}$ for which exist a $P$-martingale $\left(Z_{i}^{P}\right)$ with

$$
S_{i}^{b} \leq Z_{i}^{P} \leq S_{i}^{a}, \quad i=0, . ., N .
$$

It follows $\mathcal{Q}^{e} \subseteq \mathcal{P}$ : for each $Q^{e} \in \mathcal{Q}^{e}$ there is a $Q^{e}$-martingale $\left(Z_{i}^{Q^{e}}\right)$, namely

$$
Z_{i}^{Q^{e}}:=E_{Q^{e}}\left[S_{N} \mid \mathcal{F}_{i}\right]
$$

such that

$$
S_{i}^{b} \leq Z_{i}^{Q^{e}} \leq S_{i}^{a}, \quad i=0, . ., N .
$$

Let $C \in L^{\infty}$. By Jouini (2000, Theorem 1.), the value $p^{*}(C):=\sup _{P \in \mathcal{P}} E_{P}(C)$ is less or equal to the price of the smallest self-financing trading strategy dominating $C$. As $p_{0}(C) \leq p^{*}(C)$, we conclude.

\section{Proof of Theorem 4.1}

We first assume $C^{(N)}$ models a European contingent claim and can be described by a function $h$ as in Equation (10). Let $u_{N}, d_{N}$ and $p_{N}^{q}$ be defined as in Section 3. Then it holds for the processes describing the ask price $\left(A_{i}\right)_{i=0,1, \ldots, N}$ and the bid price $\left(B_{i}\right)_{i=0,1, \ldots, N}$ of $C^{(N)}$,

$$
A_{i}(\omega)=a_{i}\left(\left(S_{0}, S_{1}(\omega), . ., S_{i}(\omega)\right),\right.
$$

and

$$
B_{i}(\omega)=b_{i}\left(\left(S_{0}, S_{1}(\omega), . ., S_{i}(\omega)\right),\right.
$$

where the functions $a_{i}$ and $b_{i}$ are recursively defined:

$$
b_{N}\left(x_{0}, \ldots, x_{N}\right)=a_{N}\left(x_{0}, \ldots, x_{N}\right)=h\left(x_{0}, \ldots, x_{N}\right)
$$

and for $i=0,1, . ., N-1$, if the European contingent claim is increasing

$$
\begin{aligned}
a_{i}\left(x_{0}, \ldots, x_{i}\right)= & \left(1-\Psi^{\gamma_{N}}\left(p_{N}^{q}+\varphi(N)\right)\right) a_{i+1}\left(x_{0}, \ldots, x_{i}, x_{i} d_{N}\right) \\
& +\Psi^{\gamma_{N}}\left(p_{N}^{q}+\varphi(N)\right) a_{i+1}\left(x_{0}, \ldots, x_{i}, x_{i} u_{N}\right)
\end{aligned}
$$

and

$$
\begin{aligned}
b_{i}\left(x_{0}, \ldots, x_{i}\right)= & \Psi^{\gamma_{N}}\left(1-\left(p_{N}^{q}+\varphi(N)\right)\right) b_{i+1}\left(x_{0}, \ldots, x_{i}, x_{i} d_{N}\right) \\
& +\left(1-\Psi^{\gamma_{N}}\left(1-\left(p_{N}^{q}+\varphi(N)\right)\right)\right) b_{i+1}\left(x_{0}, \ldots, x_{i}, x_{i} u_{N}\right) .
\end{aligned}
$$

If $h$ defines a decreasing European contingent claim, it follows for $i=0, . ., N-1$,

$$
\begin{aligned}
a_{i}\left(x_{0}, \ldots, x_{i}\right)= & \Psi^{\gamma_{N}}\left(1-\left(p_{N}^{q}+\varphi(N)\right)\right) a_{i+1}\left(x_{0}, \ldots, x_{i}, x_{i} d_{N}\right) \\
& +\left(1-\Psi^{\gamma_{N}}\left(1-\left(p_{N}^{q}+\varphi(N)\right)\right)\right) a_{i+1}\left(x_{0}, \ldots, x_{i}, x_{i} u_{N}\right)
\end{aligned}
$$


and

$$
\begin{aligned}
b_{i}\left(x_{0}, \ldots, x_{i}\right)= & \left(1-\Psi^{\gamma_{N}}\left(p_{N}^{q}+\varphi(N)\right)\right) b_{i+1}\left(x_{0}, \ldots, x_{i}, x_{i} d_{N}\right) \\
& +\Psi^{\gamma_{N}}\left(p_{N}^{q}+\varphi(N)\right) b_{i+1}\left(x_{0}, \ldots, x_{i}, x_{i} u_{N}\right) .
\end{aligned}
$$

The ask (bid) price of an increasing European payoff and the bid (ask) price of and decreasing European payoff at level $\gamma_{N} \geq 0$ are exactly defined as the risk-neutral price in the classical binomial model, when replacing the up-move probability in Equation (8), i.e,

$$
p_{N}^{q}+\varphi(N)
$$

by

$$
\Psi^{\gamma_{N}}\left(p_{N}^{q}+\varphi(N)\right)
$$

respectively by

$$
\left(1-\Psi^{\gamma_{N}}\left(1-\left(p_{N}^{q}+\varphi(N)\right)\right)\right) .
$$

This observation can directly be carried forward to American contingent claims and is explained by the structure of the binomial model and the recursive definition of bid and ask prices.

By Assumption A2, there is a sequence $\tilde{\varphi}(N) \in o\left(\frac{1}{\sqrt{N}}\right)$ such that

$$
\begin{aligned}
\Psi^{\gamma_{N}}\left(p_{N}^{q}+\varphi(N)\right) & =\frac{1}{2}+\frac{r-q}{2 \sigma} \sqrt{\frac{T}{N}}+\frac{\gamma}{2} \sqrt{\frac{T}{N}}+\tilde{\varphi}(N) \\
& =\frac{1}{2}+\frac{r-(q-\sigma \gamma)}{2 \sigma} \sqrt{\frac{T}{N}}+\tilde{\varphi}(N) \\
& =p_{N}^{q-\sigma \gamma}+\tilde{\varphi}(N) .
\end{aligned}
$$

Similarly it holds for a suitable $\hat{\varphi}(N) \in o\left(\frac{1}{\sqrt{N}}\right)$,

$$
1-\Psi^{\gamma_{N}}\left(1-\left(p_{N}^{q}+\varphi(N)\right)\right)=p_{N}^{q+\sigma \gamma}+\hat{\varphi}(N) .
$$

Hence the up-move probability of the distorted binomial model describing bid and ask prices can be expressed as in the classical binomial model with an adjusted dividend yield:

$$
\frac{1}{2}+\frac{r-(q \pm \sigma \gamma)}{2 \sigma} \sqrt{\frac{T}{N}}+o\left(\frac{1}{\sqrt{N}}\right) .
$$

As the up and down moves $u_{N}$ and $d_{N}$ remain unchanged compared to the classical binomial model, we conclude.

\section{References}

[1] Acharya, V. V., \& Pedersen, L. H. (2005). Asset pricing with liquidity risk. Journal of financial Economics, 77(2), 375-410. 
[2] Albrecher, Hansjoerg, Florence Guillaume, and Wim Schoutens. 2013. "Implied liquidity: Model sensitivity." Journal of Empirical Finance 23: 48-67.

[3] Amin, Kaushik, and Ajay Khanna. 1994. "Convergence of American option values from discrete to continuous-time financial models." Mathematical Finance 4, no. 4: 289-304.

[4] Artzner, Philippe, Freddy Delbaen, Jean-Marc Eber, and David Heath. 1999. "Coherent measures of risk." Mathematical Finance 9, no. 3: 203228.

[5] Balbás, Alejandro, José Garrido, and Silvia Mayoral. 2009. "Properties of distortion risk measures." Methodology and Computing in Applied Probability 11, no. 3: 385 .

[6] Barone-Adesi, Giovanni, and Robert E. Whaley. 1987. "Efficient analytic approximation of American option values." The Journal of Finance 42, no. 2: $301-320$.

[7] Bensaid, Bernard, Jean-Philippe Lesne, Henri Pages, and José Scheinkman. 1992. "Derivative asset pricing with transaction costs." Mathematical Finance 2, no. 2: 63-86.

[8] Bernardo, Antonio E., and Olivier Ledoit. 2000. "Gain, loss, and asset pricing." Journal of Political Economy 108, no. 1: 144-172.

[9] Bielecki, Tomasz R., Igor Cialenco, Ismail Iyigunler, and Rodrigo Rodriguez. 2013. "Dynamic conic finance: Pricing and hedging in market models with transaction costs via dynamic coherent acceptability indices." International Journal of Theoretical and Applied Finance 16, no. 01: 1350002.

[10] Bielecki, Tomasz R., Igor Cialenco, and Tao Chen. 2015. "Dynamic conic finance via backward stochastic difference equations." SIAM Journal on Financial Mathematics 6, no. 1: 1068-1122.

[11] Bion-Nadal, Jocelyne. 2009. "Bid-ask dynamic pricing in financial markets with transaction costs and liquidity risk." Journal of Mathematical Economics 45, no. 11: 738-750.

[12] Bjerksund, Petter, and Gunnar Stensland. 1993. "Closed-form approximation of American options." Scandinavian Journal of Management 9: S87S99.

[13] Black, Fischer, and Myron Scholes. 1973. "The pricing of options and corporate liabilities." Journal of Political Economy 81, no. 3: 637-654.

[14] Boyle, Phelim P., and Ton Vorst. 1992. "Option replication in discrete time with transaction costs." The Journal of Finance 47, no. 1: 271-293.

[15] Carbone, Raffaella. 2004. "Binomial approximation of Brownian motion and its maximum." Statistics 85 Probability Letters 69, no. 3: 271-285. 
[16] Carr, Peter, Helyette Geman, and Dilip B. Madan. 2001. "Pricing and hedging in incomplete markets." Journal of Financial Economics 62, no. 1: 131-167.

[17] Cetin, Umut, Robert A. Jarrow, and Philip Protter. 2004. "Liquidity risk and arbitrage pricing theory." Finance and Stochastics 8, no. 3: 311-341.

[18] Cheng, Kevin. 2003. "An overview of barrier options." Global Derivatives.

[19] Cherny, Alexander S. 2007. "Pricing and hedging European options with discrete-time coherent risk." Finance and Stochastics 11, no. 4: 537-569.

[20] Cherny, Alexander, and Dilip Madan. 2008. "New measures for performance evaluation." The Review of Financial Studies 22, no. 7: 2571-2606.

[21] Cohen, Samuel N., and Robert J. Elliott. 2010. "A general theory of finite state backward stochastic difference equations." Stochastic Processes and their Applications 120, no. 4: 442-466.

[22] Corcuera, José Manuel, Florence Guillaume, Dilip B. Madan, and Wim Schoutens. 2012. "Implied liquidity: towards stochastic liquidity modelling and liquidity trading." International Journal of Portfolio Analysis and Management 1, no. 1: 80-91.

[23] Cox, John C., Stephen A. Ross, and Mark Rubinstein. 1979. "Option pricing: A simplified approach." Journal of Financial Economics 7, no. 3: 229263.

[24] Dhaene, Jan, J. Dony, M. B. Forys, Daniël Linders, and Wim Schoutens. 2012. "FIX: The Fear Index-Measuring Market Fear." Topics in Numerical Methods for Finance, 37-55. Springer, Boston, MA.

[25] Föllmer, Hans, and Irina Penner. 2006. "Convex risk measures and the dynamics of their penalty functions." Statistics \& Decisions 24, no. 1/2006: 61-96.

[26] Föllmer, Hans, and Alexander Schied. 2002. "Convex measures of risk and trading constraints." Finance and Stochastics 6, no. 4: 429-447.

[27] Föllmer, Hans, and Alexander Schied. 2011. Stochastic finance: an introduction in discrete time. Walter de Gruyter

[28] Goyenko, R. Y., Holden, C. W., \& Trzcinka, C. A. (2009). Do liquidity measures measure liquidity?. Journal of financial Economics, 92(2), 153181.

[29] Guillaume, Florence. 2015. "The LIX: A model-independent liquidity index." Journal of Banking \& Finance 58: 214-231. 
[30] Guillaume, Florence, Gero Junike, Peter Leoni and Wim Schoutens. 2018. Implied liquidity risk premia in option markets. Annals of Finance, https://doi.org/10.1007/s10436-018-0339-y.

[31] Guillaume, Florence, and Wim Schoutens. 2015. "Bid-Ask Spread for Exotic Options under Conic Finance." Innovations in Quantitative Risk Management, 59-74.

[32] Heston, Steve, and Guofu Zhou. 2000. "On the rate of convergence of discrete-time contingent claims." Mathematical Finance 10.1: 53-75.

[33] Jaschke, Stefan, and Uwe Küchler. 2001. "Coherent risk measures and gooddeal bounds." Finance and Stochastics 5, no. 2: 181-200.

[34] Jiang, Lishang, and Min Dai. 2004. "Convergence of binomial tree methods for European/American path-dependent options." SIAM Journal on Numerical Analysis 42, no. 3: 1094-1109.

[35] Jouini, Elyès. 2000. "Price functionals with bid-ask spreads: an axiomatic approach." Journal of Mathematical Economics 34, no. 4: 547-558.

[36] Jouini, Elyès, and Hédi Kallal. 1995. "Martingales and arbitrage in securities markets with transaction costs." Journal of Economic Theory 66, no. 1: $178-197$.

[37] Jouini, Elyès, and Hédi Kallal. "Efficient trading strategies in the presence of market frictions." The Review of Financial Studies 14, no. 2 2001: 343369 .

[38] Koehl, Pierre-F., and Huyên Pham. 2000. "Sublinear price functionals under portfolio constraints." Journal of Mathematical Economics 33, no. 3: $339-351$.

[39] Kusuoka, Shigeo. 2001 "On law invariant coherent risk measures." Advances in Mathematical Economics, 83-95. Springer, Tokyo.

[40] Lamberton, Damien. 1998. "Error estimates for the binomial approximation of American put options." The Annals of Applied Probability 8.1: 206-233.

[41] Leippold, Markus, and Steven Schärer. 2017. "Discrete-time option pricing with stochastic liquidity." Journal of Banking \& Finance 75: 1-16.

[42] Leisen, Dietmar PJ. 1998. "Pricing the American put option: A detailed convergence analysis for binomial models." Journal of Economic Dynamics and Control 22.8-9: 1419-1444.

[43] Leisen, Dietmar PJ, and Matthias Reimer. 1996. "Binomial models for option valuation-examining and improving convergence." Applied Mathematical Finance 3.4: 319-346. 
[44] Leland, Hayne E. 1985. "Option pricing and replication with transactions costs." The Journal of Finance 40, no. 5: 1283-1301.

[45] Lin, Jhihrong, and Ken Palmer. 2013. "Convergence of barrier option prices in the binomial model." Mathematical Finance: An International Journal of Mathematics, Statistics and Financial Economics 23, no. 2: 318-338.

[46] Liu, W. (2006). A liquidity-augmented capital asset pricing model. Journal of financial Economics, 82(3), 631-671.

[47] Madan, Dilip B. 2010. "Conserving capital by adjusting deltas for gamma in the presence of skewness." Journal of Risk and Financial Management 3, no. 1: 1-25.

[48] Madan, Dilip B. 2012a. "Execution costs and efficient execution frontiers." Annals of Financial Economics 7, no. 01: 1250002.

[49] Madan, Dilip B. 2012b. "On pricing contingent capital notes." Recent Advances in Financial Engineering, 21-42.

[50] Madan, Dilip B. 2014. "Modeling and monitoring risk acceptability in markets: The case of the credit default swap market." Journal of Banking $\mathscr{E}$ Finance 47: 63-73.

[51] Madan, Dilip B. 2016. "Conic portfolio theory." International Journal of Theoretical and Applied Finance 19, no. 03: 1650019.

[52] Madan, Dilip B. 2018. "Nonlinear equity valuation using conic finance and its regulatory implications." Mathematics and Financial Economics, 1-35.

[53] Madan, Dilip B., and Alexander Cherny. 2010. "Markets as a counterparty: an introduction to conic finance." International Journal of Theoretical and Applied Finance 13, no. 08: 1149-1177.

[54] Madan, Dilip B., Martijn Pistorius, and Wim Schoutens. 2013. "The valuation of structured products using Markov chain models." Quantitative Finance 13, no. 1: 125-136.

[55] Madan, Dilip B., Martijn Pistorius, and Wim Schoutens. 2016. "Dynamic conic hedging for competitiveness." Mathematics and Financial Economics 10, no. 4: 405-439.

[56] Madan, Dilip B., Martijn Pistorius, and Wim Schoutens. 2017. "Conic trading in a markovian steady state." International Journal of Theoretical and Applied Finance 20, no. 02: 1750010.

[57] Madan, D. B., M. Pistorius, and M. Stadje. 2012. "On Consistent Valuation Based on Distortions: From Multinomial Random Walks to Lévy Processes", Imperial College Preprint, Available at http://arxiv.org/abs/1301.3531. 
[58] Madan, Dilip, Martijn Pistorius, and Mitja Stadje. 2017. "On dynamic spectral risk measures, a limit theorem and optimal portfolio allocation." Finance and Stochastics 21, no. 4: 1073-1102.

[59] Madan, Dilip B., and Wim Schoutens. 2011. "Conic coconuts: the pricing of contingent capital notes using conic finance." Mathematics and Financial Economics 4, no. 2: 87-106.

[60] Madan, Dilip B., and Wim Schoutens. 2012a. "Structured products equilibria in conic two price markets." Mathematics and Financial Economics 6, no. 1: 37-57.

[61] Madan, Dilip B., and Wim Schoutens. 2012b. "Tenor specific pricing." International Journal of Theoretical and Applied Finance 15, no. 06: 1250043.

[62] Madan, Dilip, and Wim Schoutens. 2016a. Applied Conic Finance. Cambridge University Press.

[63] Madan, Dilip B., and Wim Schoutens. 2016b. "Conic CVA and DVA." Wilmott, no. 82 (2016): 45-50.

[64] Peng, Shige. 2004. "Nonlinear expectations, nonlinear evaluations and risk measures." Stochastic Methods in Finance, 165-253.

[65] Pınar, Mustafa Ç., Aslıhan Salih, and Ahmet Camcı. 2010. "Expected gain-loss pricing and hedging of contingent claims in incomplete markets by linear programming." European Journal of Operational Research 201, no. 3: $770-785$.

[66] Roorda, Berend, Johannes M. Schumacher, and Jacob Engwerda. 2005. "Coherent acceptability measures in multiperiod models." Mathematical Finance: An International Journal of Mathematics, Statistics and Financial Economics 15, no. 4: 589-612.

[67] Roux, Alet, Krzysztof Tokarz, and Tomasz Zastawniak. 2008. "Options under proportional transaction costs: An algorithmic approach to pricing and hedging." Acta Applicandae Mathematicae 103, no. 2: 201-219.

[68] Primbs, James A., and Yuji Yamada. 2008. "A new computational tool for analysing dynamic hedging under transaction costs." Quantitative Finance 8, no. 4: 405-413.

[69] Rubinstein, M. and Reiner, E. 1991. Breaking down the barriers, Risk Magazine, 48, 28-35.

[70] Soner, Halil M., Steven E. Shreve, and Jaksa Cvitanic. 1995. "There is no nontrivial hedging portfolio for option pricing with transaction costs." The Annals of Applied Probability 5, no. 2: 327-355. 
[71] Staum, Jeremy. 2004. "Fundamental theorems of asset pricing for good deal bounds." Mathematical Finance: An International Journal of Mathematics, Statistics and Financial Economics 14, no. 2: 141-161.

[72] Toft, Klaus Bjerre. 1996. "On the mean-variance tradeoff in option replication with transactions costs." Journal of Financial and Quantitative Analysis 31, no. 2: 233-263.

[73] Tokarz, Krzysztof, and Tomasz Zastawniak. 2006. "American contingent claims under small proportional transaction costs." Journal of Mathematical Economics 43, no. 1: 65-85.

[74] Wang, Shaun. 1996. "Premium calculation by transforming the layer premium density." ASTIN Bulletin: The Journal of the IAA 26, no. 1: 71-92.

[75] Wang, Shaun S. 2000. "A class of distortion operators for pricing financial and insurance risks." Journal of Risk and Insurance: 15-36. 